\title{
Analytical solution for priority based Handover voice and data packets in mobile multimedia WSN
}

\author{
[ TaimurKaramat, TehminaKaramat Khan, Jibran Khan ]
}

\begin{abstract}
In WSN's (wireless sensor networks) there are applications for which the sensor node requiresbeing mobile. To support uninterrupted service to nodes mobility plays vital role. In wireless services the blocking of an ongoing session is more objectionable than the blocking of an originating session and has diverse effect on the quality of service of the overall network. As compared to static WSN networks mobile WSN networks require high data quality. Hence important of all is QoS, which includes but not limited to hand over drop out packets for voice and data packets/calls, packet loss due to time out periods, reliable data transfer, efficient transmission, minimum error rate (to reduce retransmission of packets)etc. In this paper in order to minimize the drop out hand over voice and data packets and packet loss we had devised a priority based analytical solution where hand over voice and data packets/packets are prioritize. Analytical solution devise do saves computational power, human resource and is generic in nature; is robust and can be reused with additional or reduce parameters as compared to computational solutions.
\end{abstract}

Keywords-Wireless sensor networks,HO(Hand over), mobility.

\section{Introduction}

Wireless senor networks have been developed rapidly in recent years due to its capabilities of independent computing, data communication and sensing, and long battery life and low cost deployment [3]. The sensor networks do not require expansive equipment to manufacture them. In many WSN's applications sensor nodes require to be mobile. Several issues need to be maintained and handled i.e. auto configuration of networks, hand off, security, addressing routing and $\mathrm{HO}$ (Hand over) to support mobility.

In this paper the focus is on $\mathrm{HO}$ (Hand over) voice packets and hand off Data packets service and by analyzing the performance of the sensor network it may be possible that the issues involved in the hand off 's are not only be identified but also on the basis of the findings through this paper suggestions can be given to eradicate the problems in future research work. The main problem which will be

Taimur Karamat

Virtual University of Pakistan,

Lawrence Road Lahore

Pakistan

TehminaKaramat Khan

$\mathrm{PhD}$ scholar

International Islamic University

Malaysia

Jibran Khan

Virtual University of Pakistan

Peshawar Campus, KPKPakistan researched through this paper is blocking and waiting time's of hand off voice and data packets also will observe the pattern of arrival of hand off packets, it will research that if the packet is dropped then it what could be a possible reason whether it is due to buffer being full or service problem in the server. This will not only try to suggest future work towards the retrieving of dropped hand off's for voice and data but may also suggest virtual buffering/Queuing mechanism for future work and measure to avoid the call deletion.

\section{A. Related Work}

There have been a lot of work done in diverse directions to improve QoS of WSN networks, by improving routing protocols, by removing un necessary time for hand over packets/calls, by introducing low power sensor's, other techniques are but not limited to are RI-MAC(Receiverinitiated MAC): the protocol is used for broadcasting and during data transmission it degrade the link quality by searching a new relay node without interrupting previous nodes communication, by Beacon Enabled IEEE 802.11.4 mac: the protocol uses two methods to transmit data which are beacon enabled and non beacon enabled mode, Handover based on 6LoWPAN AND Soft handover based on 6LoWPAN, by GT-MOTS(game theory inspired mobile object trapping system) the protocol exploit the WSN in the form objects it is a part of a game theory [3]. In [1] the author has presented statistical routing protocol which is a solution to overcome the shot comings in traditional hand over process, also the author has suggested ways on how the statistical routing be considered as an alternative to traditional routing protocol in WSN where capabilities of the nodes are limited, in [2] challenges in mobility management are identified and the author has presented with possible solution for handle mobility management such as LoWPAN6 (Low Power Sensor). In [3] a single buffer solution to handle hand over voice and hand over data call is presented, calls do enter the system when channels are available to process calls but in case all channels remainbusy processing calls HO voice calls start entering buffer and hand over data calls are blocked, when the buffer is full the hand over voice calls are blocked as well, in case channel is available the hand over calls succeed. In [7] a protocol RIMAC is proposed for theprovisioning of seamless handover. In this scenario the momentMN(mobile node) recognizes that the data packet can't be transmittedcompletely before breakage ofexisting link, it will look for fresh relay node besides the communication interruption with current node. In this paper a mathematical model is set up for latency investigation because of handover $(\mathrm{HO})$. The analytical model enumerates the HO latency as a function of data cycle and network density. This paper removes the drawback of RI-MAC i.e. BW(back off window field) size it introduces 
bursty transmission form of data packets thus rather than competing for media for sending single data packet, single node transmit on a predefined number of data packets in burst which might be unfair, but in terms of energy consumption and latency it is efficient.

All work done is to improve the QoS, in our paper we had proposed an analytical model and performance evaluation of a WSN network is carried out in terms of mean waiting time, mean number of packets and blocking of hand over voice and data packets, by assigning dedicated channels for hand over voice and data packets, and shared channels for originating voice and data packets. Also in our model we have buffer for hand over voice packets and for hand over data packets. The numerical results shows that the QoS i.e. waiting time $\mathrm{W}$ and blocking can be reduced by increasing the number of shared channels.

\section{Model Assumptions}

In this model we have total of $\mathrm{X}$ channels, with parameter $\mu$ withchannels holding time exponentially distributed, i.e. service timewith mean $1 / \mu$ is independently distributed. The model diagram is shown in fig $1.1 \mathrm{a}$

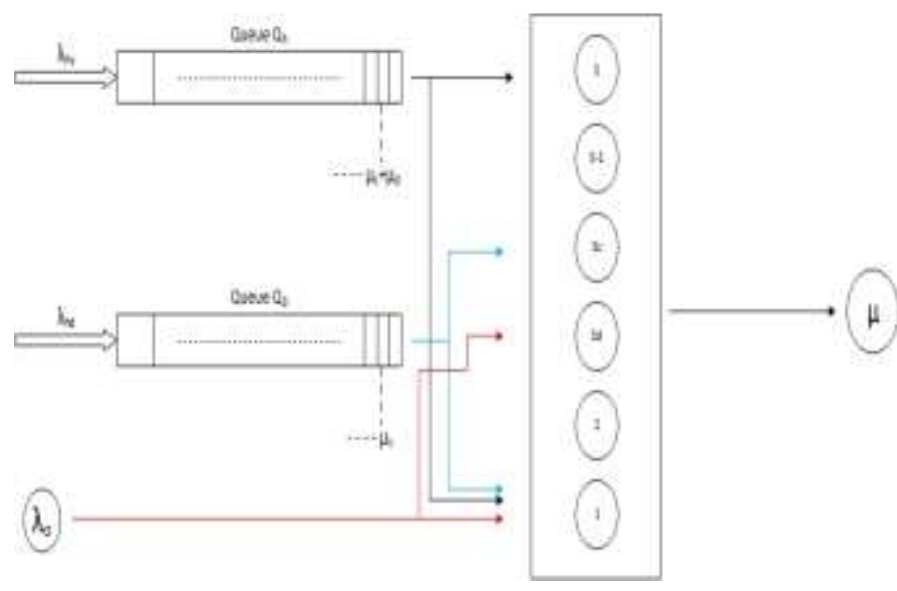

Fig 1.1a

There are four type of packets i.e. originating voice packets, originating data packets, $\mathrm{HO}$ (Hand over) voice packets and $\mathrm{HO}$ (Hand over) data packets. Let the four random variables for inter arrival time of $\mathrm{HO}$ (hand over) voice, HO data, originating voice and originating data be $L_{o d}, L_{o v}, L_{h d}$ and $L_{h v}$, the arrival of packets occurs in accordance with poison process from infinite source with parameter $\lambda_{o d c}, \lambda_{o v c}, \lambda_{h d c}$ and $\lambda_{h v c}$ respectively, all the inter arrival timeswith mean $\frac{1}{\lambda_{o d c}}, \frac{1}{\lambda_{o v c}}, \frac{1}{\lambda_{h d c}}$ and $\frac{1}{\lambda_{h v c}}$ areindependent and exponentially distributed. Therefore by memoryless property of exponential distribution remaining inter arrival time i.e. $\Delta L_{o d}, \Delta L_{o v}, \Delta L_{h d}$ and $\Delta L_{h v}$ is also exponentially distributed, by parameter $\Delta \lambda_{o d c}, \Delta \lambda_{o v c}$, $\Delta \lambda_{h d c}$ and $\Delta \lambda_{h v c}$. Next arrival we can say will be minimum( $\left.\Delta L_{o d}, \Delta L_{o v}, \Delta L_{h d}, \Delta L_{h v}\right)$ [2]. Next arrival therefore will be exponentially distributed by parameter $1 / \lambda$ i.e. summation of $\lambda_{o d c}, \lambda_{o v c}, \lambda_{h d c}$ and $\lambda_{h v c}$ given by

$$
\lambda=\lambda_{o d c}+\lambda_{o v c}+\lambda_{h d c}+\lambda_{h v c}
$$

In case $\mathrm{X}_{c}$ channels are processing packets originating data and voice packets will be blocked andin case $\mathrm{X}_{d}$ channels become busy processing packets hand off data packets are queued in queuesized $\mathrm{N}_{o}$, when queue is full hand off data packets will get blocked. Blocked packets are lost and do not return to system. A HO(Hand over) data packet is deleted from queue in case it moves out of cell before get served, rate of these packets is $\mu_{t}$ and are exponentially distributed bymean $1 / \mu_{a}$. TheHO(Hand over) voice packets get service when any of the $S$ channels is free for service, $\mathrm{HO}$ (Hand over) voice packets on arrival when finds all $\mathrm{X}$ channels busy processing are queued in queue withlength $N_{h}$; in case the queue is fullHO(Hand over)voice packets are lost and the lost packets leave immediately and does not return to the system.

$$
\begin{array}{ll}
\lambda=\lambda_{o d c}+\lambda_{o v c}+\lambda_{h d c}+\lambda_{h v c} & 0 \leq m_{1} \leq X_{c} \\
\lambda^{\prime}=\lambda_{h d c}+\lambda_{h v c} & X_{c}<m_{1}<X_{d} \\
\lambda=\lambda_{h v c} & X_{d} \leq m_{1} \leq X+N_{h} \\
\lambda=\lambda_{h d c} \quad 1 \leq m_{2} \leq N_{o}-1
\end{array}
$$

Else 0

Some of $\mathrm{HO}$ (Hand over) voice packets waiting in queue may get dropped out. There are two reasons for that either it's because the session get completed or because of the time out period. The packet that get dropped because of session completion is calledsmooth termination;and is exponentially distributed with mean $1 / \mu_{b}$. The packet that get dropped because of time out period is called force termination and is exponentially distributed with mean $1 / \mu_{e}$

The parameters $1 / \mu_{b}, 1 / \mu_{e}$ and $1 / \mu_{a}$ are exponentially distributed, the remaining time $\Delta \mu_{b}, \Delta \mu_{e}$ and $\Delta \mu$ are also exponentially distributed consequently next departure is min $\left(\Delta \mu_{b}, \Delta \mu_{e} \Delta \mu\right)$. Service time is exponentially distributed as per memory less property of exponential distribution with mean “ $\frac{1}{\mu_{b},+\mu_{e}+\mu}$ "

$$
\begin{array}{cc}
\mu_{m 1}=m_{1} \mu & 1 \leq m_{1} \leq \mathrm{X} \\
=\mathrm{X} \mu+m_{1}\left(\mu_{b}+\mu_{e}\right) & \mathrm{X}<m_{1} \leq X+N_{h} \\
\mu_{m_{2}}=X_{c} \mu+m_{2} \mu_{a} & 1 \leq m_{2} \leq X_{o} \\
m_{2}=X_{c} \\
X_{c}<m_{1} \leq X+N_{h} \\
m_{2} \mu_{a} & 1 \leq m_{1} \leq N_{o}
\end{array}
$$

Else 0

State transition diagram is shown in fig.1a 
Proc. of the Fourth Intl. Conf. Advances in Computing, Communication and Information Technology- CCIT 2016 Copyright (C) Institute of Research Engineers and Doctors, USA .All rights reserved.

ISBN: 978-1-63248-092-7 doi: 10.15224/ 978-1-63248-092-7-13

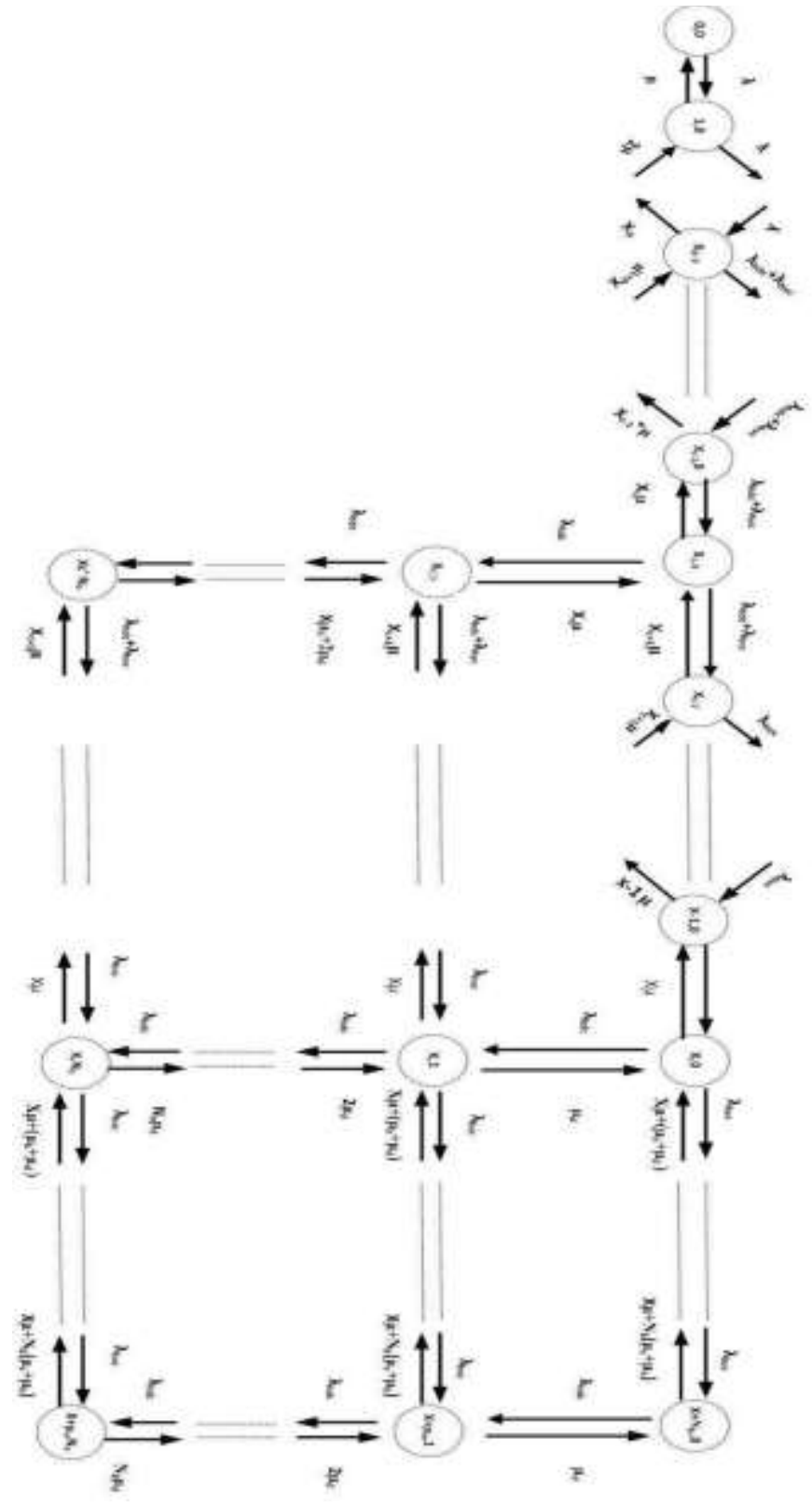

Fig. 1a

It has been supposed that the system is in steady state, therefore for each state $\mathrm{G}\left(m_{1}, m_{2}\right)\left(m_{1}=0,1,2,3 \ldots \ldots N_{h}\right.$, $n_{2}=0,1,2,3 \ldots \ldots N_{o}$ ) mean flow into a state equates equal to mean flow out of that state. To calculate performance metrics flow balance equations is the base in queueing system.

Flow rate out of state $\mathrm{G}\left(m_{1}, m_{2}\right)$ is equated to flow rate into state $\mathrm{G}\left(m_{1}, m_{2}\right)$, we get equations at equilibrium for the state probabilities $\mathrm{p}\left(m_{1}, m_{2}\right)$. When the rates are substituted, state probabilities $p\left(m_{1}, m_{2}\right)$ exhibit the product form as in L-Rule in [10]

$$
p\left(m_{1}, m_{2}\right)=C * \frac{\rho_{1} m_{1} \rho_{2} m_{2}}{m_{1} ! m_{2} !}
$$

In the equation above $\mathrm{C}$ is constant, now let suppose $m_{1}=m_{2}=0$, above equation will be of form

$$
p(0,0)=C
$$

Hence

$$
p\left(m_{1}, m_{2}\right)=\frac{\rho_{1}{ }^{m_{1}} \rho_{2}{ }^{m_{2}}}{m_{1} ! m_{2} !} p(0,0)
$$

Summation of probabilities equates to 1 , Therefore

$$
\begin{aligned}
& {\left[1+\sum_{m 1=1}^{X_{d}-1} \frac{\rho^{m 1}}{m_{1} !}+\sum_{m 1=X_{d}}^{X c} \frac{\lambda^{X_{d}} \lambda^{m_{1}-X_{d}}}{m_{1} ! \mu^{m_{1}}}\right.} \\
& +\sum_{\substack{m_{2}=1 \\
m_{1}=X_{c}}}^{N_{o}-1} \frac{\lambda^{X_{d}} \lambda^{X^{X_{c}-X_{d}}} \lambda_{h d}^{m_{2}}}{X_{c} ! \mu^{X_{c}} \prod_{m=1}^{m_{2}}\left(X_{c} \mu+m \mu_{d}\right)}
\end{aligned}
$$

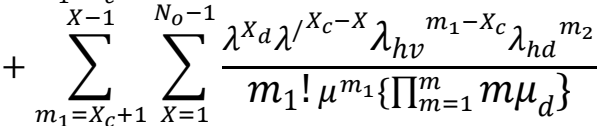

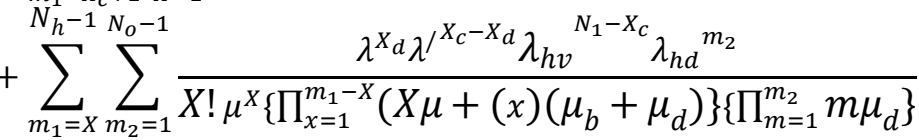

$$
\begin{aligned}
& +\sum_{\substack{m_{1}=X_{c}+1 \\
m_{2}=N_{o}}}^{X} \frac{\lambda^{X_{d} \lambda^{\prime}}{ }^{X_{C}-X_{d}} \lambda_{h v}^{m_{1}-X_{c}} \lambda_{h d}^{N_{o}}}{m ! \mu^{m_{1}} \prod_{m=1}^{N_{o}} m \mu_{d}}
\end{aligned}
$$

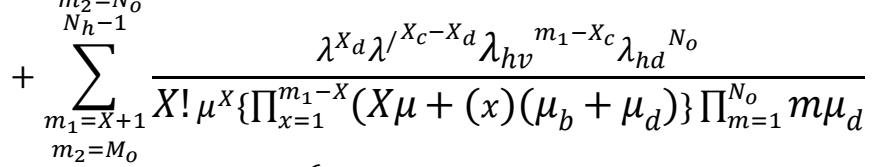

$$
\begin{aligned}
& +\sum_{\substack{m_{1}=N_{c}+1 \\
m_{2}=0}}^{X} \frac{6}{\lambda^{X_{d}} \lambda^{X^{X_{c}}-X_{d}} \lambda_{h v}^{m_{1}-X_{c}}} \\
& +\sum_{\substack{m_{1}=X+1 \\
m_{2}=0}}^{N_{h}-1} \frac{\lambda^{X_{d} \lambda^{\prime}}{ }^{X_{c}-X_{d}} \lambda_{h v}{ }^{m_{1}-X_{c}}}{X ! \mu^{X}\left\{\prod_{x=1}^{m_{1}-X}\left(X \mu+(x)\left(\mu_{b}+\mu_{d}\right)\right\}\right.} \\
& \left.+\frac{\lambda^{X_{d}} \lambda^{X^{X_{c}-X_{d}}} \lambda_{h v}{ }^{N_{h}-X_{c}} \lambda_{h d}{ }^{N_{o}}}{X ! \mu^{X}\left\{\prod_{x=1}^{N_{h}-X}\left(X \mu+(x)\left(\mu_{b}+\mu_{d}\right)\right\} \prod_{m=1}^{N_{o}} m \mu_{d}\right.}\right]^{-1} \\
& =p(0,0)
\end{aligned}
$$

\section{Performance Measures}

Mean number of packets in system, $\mathrm{L}=\sum_{m_{1}=1}^{X_{c}-1} x_{1} *$

$$
p\left(x_{1}, 0\right)+\sum_{m_{1}=S_{c}}^{N_{h}} n_{1} * \sum_{m_{2}=1}^{N_{o}} m_{2} * p\left(m_{1}, m_{2}\right)
$$

Mean number of $\mathrm{HO}$ (Hand over) data packets in Queue,

$L_{o}=\sum_{m_{1}=X_{c}}^{N_{h}} \sum_{m_{2}=1}^{N_{o}} m_{2} * p\left(m_{1}, m_{2}\right)$

Mean number of $\mathrm{HO}$ (Hand over) voice packets in Queue,

$L_{h}=\sum_{m_{1}=x+1}^{N_{h}}\left(m_{1}-X\right) * \sum_{m_{2}=1}^{N_{o}} p\left(m_{1}, m_{2}\right)$ 
Blocking probability of $\mathrm{HO}($ Hand over $)$ voice packets, $B_{O}=$

$\sum_{m_{1}=X_{c}}^{N_{h}} p\left(m_{1}, N_{o}\right)$

Blocking probability of $\mathrm{HO}$ (Hand over) voice packets, $B_{h}=$

$\sum_{m_{2}=1}^{N_{o}} p\left(N_{h}, m_{2}\right)$

Blocking probability of originating voice and data packets =

$B_{\text {ovd }}=\left\{\sum_{m_{1}=X_{d}}^{X_{c}-1} m_{1} * p\left(m_{1}, 0\right)+\sum_{m_{1}=X_{c}}^{N_{h}} m_{1} *\right.$

$\left.\sum_{m_{2}=1}^{N_{o}} m_{2} * p\left(m_{1}, m_{2}\right)\right\}$

Actual Arrival rate $=\left(\lambda_{o d c}+\lambda_{o v c}\right)\left(1-B_{o v d}\right)+$

$\lambda_{h v c} *\left(1-B_{h}\right)+\lambda_{h d c} *\left(1-B_{o}\right)$

Mean waiting time of customers, $\mathrm{W}=$ Total

customers/Actual arrival rate

Mean waiting time of $\mathrm{HO}($ Hand over) data packet in queue,

$$
W_{O C}=\frac{L_{o}}{\text { Acutal arrival rate of HO data call }}
$$

$=\frac{\left\{\sum_{\boldsymbol{m}_{\mathbf{1}}=\boldsymbol{X}_{\boldsymbol{c}}}^{N_{\boldsymbol{c}}} \sum_{\boldsymbol{m}_{\mathbf{2}}=\mathbf{1}}^{\boldsymbol{N}_{\boldsymbol{o}}} \boldsymbol{m}_{\mathbf{2}} * \boldsymbol{p}\left(\boldsymbol{m}_{\mathbf{1}}, \boldsymbol{m}_{\mathbf{2}}\right)\right\}}{\lambda_{h d c} *\left(1-\boldsymbol{B}_{\boldsymbol{o}}\right)}$

Mean waiting time of $\mathrm{HO}$ (Hand over) voice packets in queue, $W_{h c}=\frac{L_{h}}{\text { Acutal arrival rate of HO voice call }}$

$$
=\frac{\left\{\sum_{\boldsymbol{m}_{\mathbf{1}}=\boldsymbol{X}+\mathbf{1}}^{N_{h}} \sum_{\boldsymbol{m}_{\mathbf{2}}=\mathbf{1}}^{N_{\boldsymbol{o}}}\left(\boldsymbol{m}_{\mathbf{1}}-\boldsymbol{X}\right) * \boldsymbol{p}\left(\boldsymbol{m}_{\mathbf{1}}, \boldsymbol{m}_{\mathbf{2}}\right)\right\}}{\lambda_{h v c} *\left(1-\boldsymbol{B}_{\boldsymbol{h}}\right)}
$$

\section{Results}

The numerical results for mean number of packets, mean waiting time and probability of blocking for $\mathrm{HO}$ voice and data packets are shown in fig.1, fig. 2 and fig.3.

In fig. 1 below mean number of $\mathrm{HO}$ voice $\&$ data packets are compared. The number of data packets in system decrease with increase in number of shared channels and vice versa. For the voice packets these will also decrease as for shared channels all types of packets are allowed to get processed whereas in dedicated channels only $\mathrm{HO}$ packets for voice and data get processed. In caseall channels remain busy processing, only $\mathrm{HO}$ voice packets get processed till all channels are occupied.

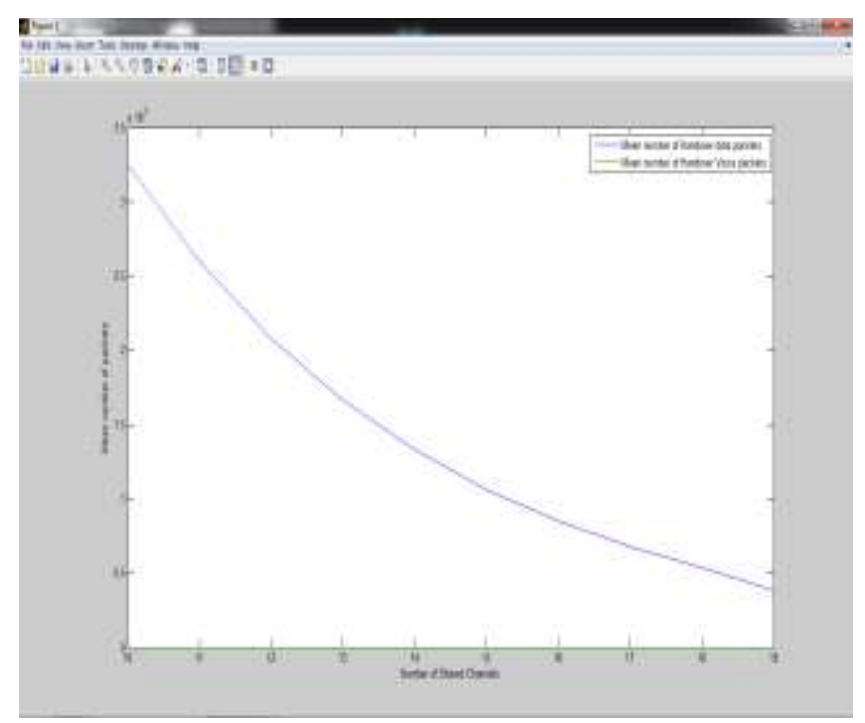

Fig.1

Mean waiting time for hand over voice and data packets are shown in fig.2. It has been observed that as the number of shared channels are increased the mean waiting time of packets do decrease as well. We can also notice that the mean waiting time for handover voice packets is nearly zero, as there are dedicated channels available for hand over voice packets only.

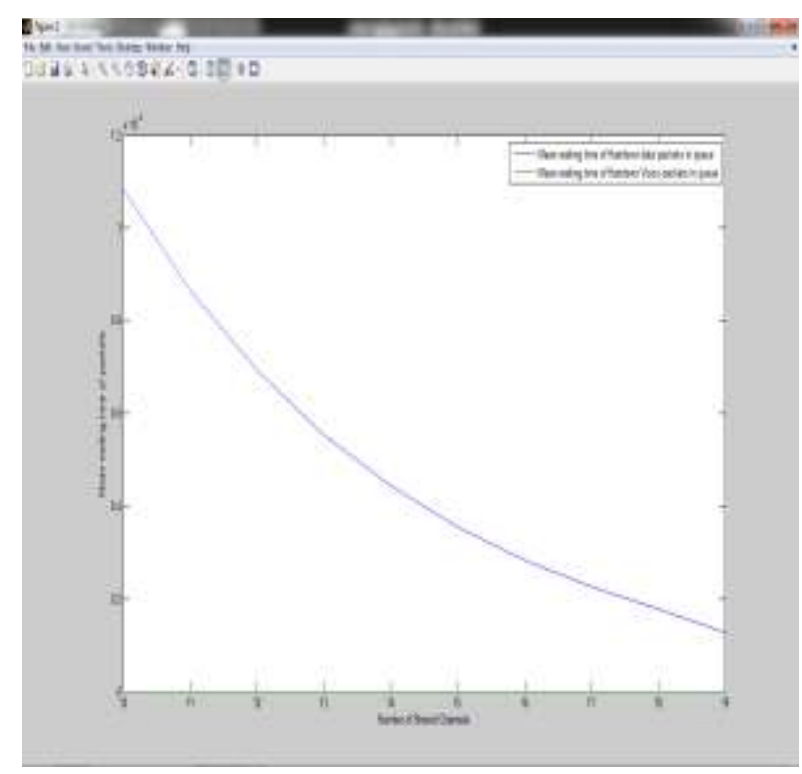

Fig.2

In fig.3, blocking probabilities of handover voice and data packets has been compared. It is obvious from the figure that the blocking probability do decrease with the increase in shared channels and vice versa. The packets that find all channels busy are blocked. The blocked packets are lost and will not return again to system. 
Proc. of the Fourth Intl. Conf. Advances in Computing, Communication and Information Technology- CCIT 2016

Copyright (c) Institute of Research Engineers and Doctors, USA .All rights reserved.

ISBN: 978-1-63248-092-7 doi: 10.15224/ 978-1-63248-092-7-13

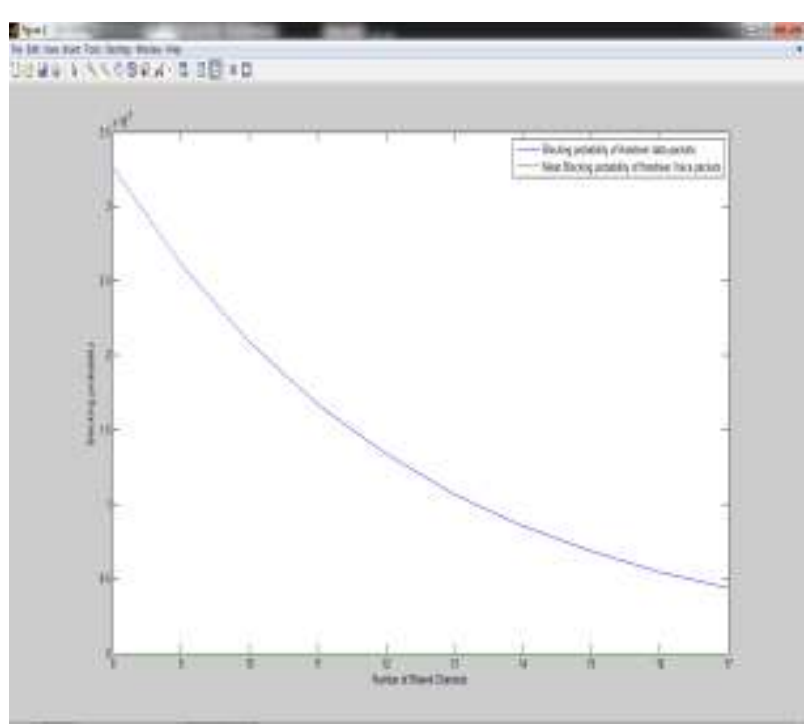

Fig.3
Communications, Distributed Computing in Sensor Systems and Workshops (DCOSS), 2011.

[9]Holge Karl, Andreas willig"ProtocolsAnd Architectures for Wireless sensor networks" John Wileys and sons Ltd. 2007

[10] Henk C. Tijms,"A first course in stochstic Models",second edition,John Wiley and sons 2003.

[11] Karamat, T, Karamat Taimur, Algorithm for seamlesshandover between WiMax and Wlan radio access technology using NS2" International Journal of Latest Research in Science and Technology ISSN (Online):2278-5299 Volume 4, Issue 1: Page No.138-141, January-February 2015.

[12] Karamat, Tc., Karamat Khan, T., "Maximum Entropy formalism of Multi Traffic Censored priority Queue" the $8^{\text {th }}$ IEEE International Symposium on Performance Modelling and Evaluation Of Computer and Telecommunication Networks ,27-29 August PMECT 2014 Barcelona Spain

[13] Karamat, Tehmina.,Karamat Khan, T., “ An Analytical Solution for a Non-Pre-emptive HO(Hand over) Priority-based Cellular Scheme with Drop out Voice and Data Calls",@ International Journal of Computer Applications (IJCA) August 2013 Edition

[14] Karamat, Tehmina.,Karamat Khan, T., "Maximum Entropy Analysis of Priority Censored LossSystem”, @ICWN, 1-2 September 2012, Phuket, Thailand

[15]Karamat, Taimur(Kouvatsos, Demetres). "Performance Evaluation and Prediction of 2-D Markovian and Bursty Multi-Traffic Queues. Analytical Solution for 2-D Markovian and Bursty Multi-Traffic Non Priority, Priority and Hand Off Calling Schemes.", University of Bradford, 2011.

channels to hand over packets we can reduce the number packets being blocked and we can also improve the performance. Because as we increase the number of shared channels mean number of packets decrease, mean waiting time each customer observe also decreases and eventually the mean waiting time decreases as well.

It will be interesting to see how the given prototype works well when the arrival and departure of packets is in burst.

There is also a possibility to do the percentage performance evaluation of the said model to improve QoS and maximizing utilization of the system.

\section{References}

[1] Siyang Shan ,Chong Shen ,Yong Bai "Statistical routing protocol for handover management in Wireless Sensor Networks" Wireless communication and applications (ICWCA)2012.

[2] Aggregate Finite-sourse modeling for multimedia communication systems",VTC,98,vol3,pp.1894-1898,1998.

[3] Jeongbae Yun, Murad Khan, and Kijun Han "A Fast HO(Hand over) Scheme for Streaming Service in Wireless Sensor Networks", International Journal of Distributed Sensor NetworksVolume 2015 (2015), Article ID 183802.

[4] Er. Taranjeet Kaur, Er. Ankush Sharma"Study of various Handover techniques using MWSN".International Journal of Computer Science and Mobile Computing, Vol.4 Issue.5, May- 2015, pg. 635-638.

[5] Waltenegus Dargie "A medium access control protocol that supports a seamless handover in wireless sensor networks" Journal of Network and Computer Applications 2011 Elsevier.

[6] Ms. S. Vinothini , Mr. V. Thiruppathy Kesavan , Ms. M. Rethina Kumari "Secure HO(Hand over) Management in Wireless Sensor Networks"International Journal of Emerging Engineering Research and Technology Volume 3, Issue 5, May 2015, PP 33-37 ISSN 23494395 (Print) \& ISSN 2349-4409

[7]Qian Dong and Waltenegus Dargie "Performance Analysis of a Handover Mechanism for a Mobile Wireless Sensor Network". The $10^{\text {th }}$ Annual IEEECCNC 2013.

[8]JuhaPetäjäjärviandHeikkiKarvonen "Soft Handover Method for Mobile Wireless Sensor Networks Based on 6LoWPAN "Centre for Wireless

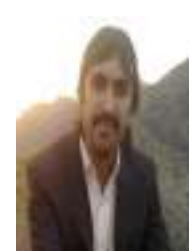

TehminaKaramat is working as Assistant Professor at foundation University Islamabad Pakistan, and $\mathrm{PhD}$ scholar at international Islamic University Malaysia. Research areas are routing and queue management of wireless networks based on queuing theoretic concepts and information theoretic principles of maximum entropy.

Jibran Khan has obtained his Master degree in computer science from department of computer science, University of Peshawar, Pakistan. Currently he is working as Tutor/Instructor at Virtual University of Pakistan and has deep interest in the field of networking and is currently working in many areas such as wireless networking, cryptography and network trafficking. 